\title{
Algorithms for Coupled Domain MEMS Simulation
}

\author{
N. Aluru and J. White \\ Research Laboratory of Electronics \\ Massachusetts Institute of Technology \\ Cambridge, MA 02139
}

\begin{abstract}
The performance of micro-electro-mechanical systems depends on the interaction between electrical, mechanical, and fluidic forces. Simulating this coupled problem is made more difficult by the fact that most MEMS devices are innately threedimensional and geometrically complicated. It is possible to simulate efficiently these devices using domain-specific solvers, provided the coupling between domains can be handled effectively. In this paper we will survey recent developments in coupled-domain simulation, and give computational comparisons between relaxation, multi-level Newton, and Newton-Iterative methods for 3-D electromechanical analysis.
\end{abstract}

\section{INTRODUCTION}

Because of the specialized processing involved, the cost of prototyping even simple microsensors, microvalves, and microactuators is enormous. In order to reduce the number of prototype failures, designers of these devices make frequent use of finite-element based simulation tools. Finite-element techniques are very general, but are very inefficient when used to simulate three-dimensional geometricallycomplicated micromechanical structures, where device performance is critically dependent on the interaction between electrostatic, mechanical and fluidic forces. Simulation efficiency can be substantially improved by using domain-specific solvers, provided the coupling between domains can be handled effectively. In this paper, we will survey recent developments in coupled-domain simulation, and give computational comparisons between relaxation, multi-level Newton, and Newton-Iterative methods for 3-D electromechanical analysis.

\section{Elastostatics And Electrostatics.}

Micro-mechanical structures undergo large deformation when subjected to electrostatic forces. This structural deformation can be determined by solving a nonlinear force balance equation which includes the material stresses and the electrostatic pressures. In particular, standard continuum mechanical analysis [6] leads to a system of partial differential equations of the form

$$
\begin{aligned}
\nabla \bullet(S(\nabla u(x))) & =0 \text { in } \Omega \\
u & =0 \text { on } \Gamma_{g} \\
n \dot{\nabla} \bullet(S(\nabla u(x))) & =\text { o on } \Gamma_{h_{i}} \\
n \times \nabla \bullet(S(\nabla u(x))) & =0 \text { on } \Gamma_{h_{i}}
\end{aligned}
$$

This research was supported by ARPA under ONR contract DABT63-94-C-0053 and FBI contract J-FBI-92-196, by SRC under contract SJ-558, and by grants from IBM and Digital Equipment Corporation.

"Permission to make digital/hard copy of all or part of this work for personal or classroom use is granted without fee provided that copies are not made or distributed for profit or commercial advantage, the copyright notice, the title of the publication and its date appear, and notice is given that copying is by permission of ACM, Inc. To copy otherwise, to republish, to post on servers or to redistribute to lists, requires prior specific permission and/or a fee."

DAC 97, Anaheim, California

(c) 1997 ACM 0-89791-920-3/97/06..\$3.50 where $x$ is a point on the initial structure, $u(x)$ is the displacement of that point from its initial position, $S$ is the nonlinear algebraic operator which relates the displacement gradient to the material stresses, $\Omega$ is the interior of the structure, $\Gamma_{g}$ is that part of the structure surface whose position is fixed, $\Gamma_{h_{i}}$ is the movable part of the structure surface which is acted on only by electrostatic pressure forces $p$.

The electrostatic pressure, $p$, on micro-mechanical conductors is related to the surface charge density, $\sigma$, as in $p=\frac{\epsilon}{2} \sigma^{2}$, where $\epsilon$ is the dielectric permittivity. This surface charge density can be determined by solving the integral equation

$$
\psi(x)=\int_{\text {surfaces }} \sigma\left(x^{\prime}\right) \frac{1}{4 \pi \epsilon_{0}\left\|x-x^{\prime}\right\|} d a^{\prime}, \quad x \in \text { surfaces }
$$

where $\psi(x)$ is the known conductor surface potential, $\sigma$ is the surface charge density, $d a^{\prime}$ is the incremental conductor surface area, $x, x^{\prime} \in \mathbf{R}^{3}$, and $\|x\|$ is the usual Euclidean length of $x$ given by $\sqrt{x_{1}^{2}+x_{2}^{2}+x_{3}^{2}}[9]$.

The most commonly used approach to solving (1) are the finiteelement methods [3]. Finite-element methods can also be used to solve a partial differential equation form of (5), and is a commonly used approach to solving the coupled electromechanical problem. However, for very complicated three-dimensional geometries, the electrostatic pressures can be computed much more efficiently using multipole or precorrected-FFT accelerated iterative methods applied directly to (5) [7], [8]. The difficulty addressed in this paper is finding approaches which lets one use the most efficient algorithm for each of the domains but still allows one to solve the coupled problem.

\section{COUPLED Methods}

Boundary-element discretization of (5) leads to a system of equations of the form

$$
P(u+x) q-\psi=R_{E}(u, q)=0
$$

where $n$ is the number of discretization unknowns, $q \in R^{n}$ is a vector of surface charges, $u+x \in R^{3 n}$ is the vector of absolute surface positions, and $P(u+x) \in R^{n \times n}$ is a dense geometry dependent matrix which relates discretized surface charges to discretized surface potentials. Here, $R_{E}$ is our notation for the electrostatic equilibrium equations, note that it indicates the dependence on geometry.

Finite-element discretization of (1) leads to a nonlinear system of equations of the form

$$
F_{M}(u)-F_{E}(q)=R_{M}(u, q)=0
$$

where $m$ is the number of discretization nodes, $u \in R^{3 m}$ is the vector of discretized node displacements, $F_{M}(u) \in R^{3 m}$ is a vector of integrated forces due to material displacement, and $F_{E}$ is the vector of integrated electrostatic pressure forces. Here, $R_{M}$ is our notation for the elastostatic equilibrium equations, note that it indicates the dependence on electrostatic forces. 


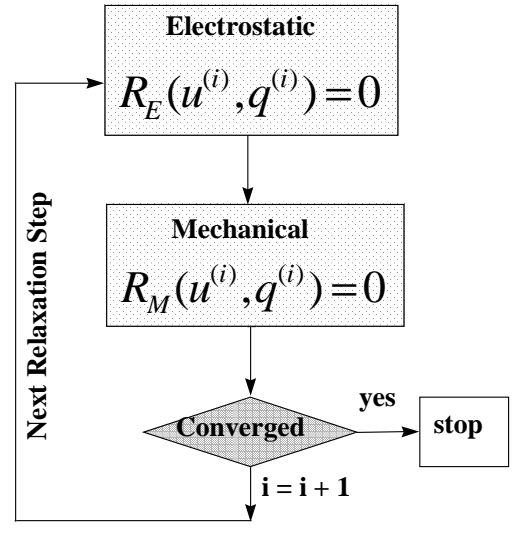

Fig. 1. A simple relaxation scheme for self-consistent electromechanical analysis.

The most obvious approach to solving the coupled system of (6) and (7) is to use a simple relaxation scheme [4], [5], [11], as diagrammed in Figure 1. The relaxation algorithm does not always converge, however, particularly when the electrostatic forces are large and the structure is extremely compliant [12].

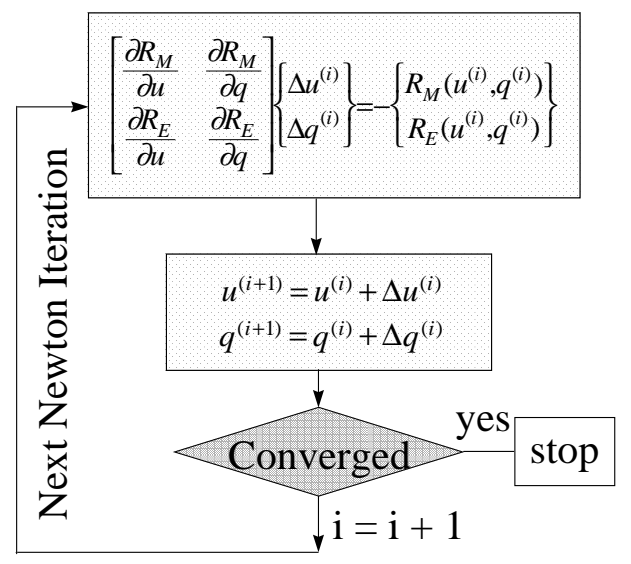

Fig. 2. A coupled algorithm for self-consistent electro-mechanical analysis.

A more robust approach is to use a full Newton algorithm to solve the coupled system, as diagrammed in Figure 2 [1]. The difficulty with the coupled approach is that the off-diagonal or coupling derivatives, $\frac{\partial R_{M}}{\partial q}$ and $\frac{\partial R_{E}}{\partial u}$ in the linear system of Figure 2, may not be available explicitly. If an iterative method, like GMRES [10], is used to solve the linear system, then only matrix-vector products are required. Therefore, the off-diagonal derivatives need not be explicitly computed, and can be approximated by finite-differences. For example, if $\Delta u$ is a part of the vector generated by GMRES, then

$$
\frac{\partial R_{E}(u, q)}{\partial u} \Delta u \approx R_{E}(u+\Delta u, q)-R_{E}(u, q)
$$

It should be noted that if Krylov-subspace methods like GMRES are used to solve the system in Figure 2, then as much of the diagonal blocks as is possible should be explicitly factored and used as a preconditioner [1]. Otherwise, the GMRES algorithm will converge too slowly to be practical.

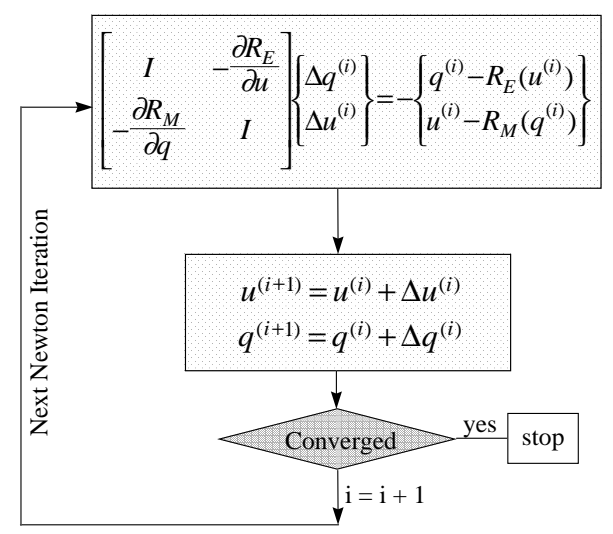

Fig. 3. A multi-level Newton algorithm for self-consistent electromechanical analysis.

Preconditioning the linear system of Figure 2 requires modifying the individual solvers somewhat. Therefore, the coupled method is not really a "black box" approach in which different domain solvers can be swapped in and out. Consider instead that a program which solves (6) can be thought of as producing charges given geometric displacments, and we will denote this as

$$
q=R_{E}(u) \text {. }
$$

In addition, a program which solves (7) can be thought of as producing geometric displacments given charges, and we denote that as

$$
u=R_{M}(q) .
$$

A multilevel-Newton method, given in Figure 3, can be used to determine the solution to the coupled system. We refer to this as a multilevel Newton method because application of the $R$ operators implies solving systems of equations, typically with an inner Newton's method. Note that in the multilevel-Newton method, the block diagonals which are already identity matrices and need not be preconditioned. Also, application of $\frac{\partial R_{E}}{\partial u}$ and $\frac{\partial R_{M}}{\partial q}$ to a vector can be performed using finite-differences, and therefore does not require modifying the domain-specific solvers.

\section{RESULTS}

Numerical results are presented for two examples: a beam over a ground plane and a comb drive structure. The performance of the relaxation, multi-level Newton and coupled algorithms is examined for both the examples. In particular, the convergence characteristics and the simulation times are compared.

\section{A. Beam Example}

The beam example considered here is $500 \mu \mathrm{m}$ long, $50 \mu \mathrm{m}$ wide, $14.35 \mu \mathrm{m}$ thick and is positioned $1 \mu \mathrm{m}$ above the ground plane. Figure 4 shows a top view of the beam example. The beam is discretized 


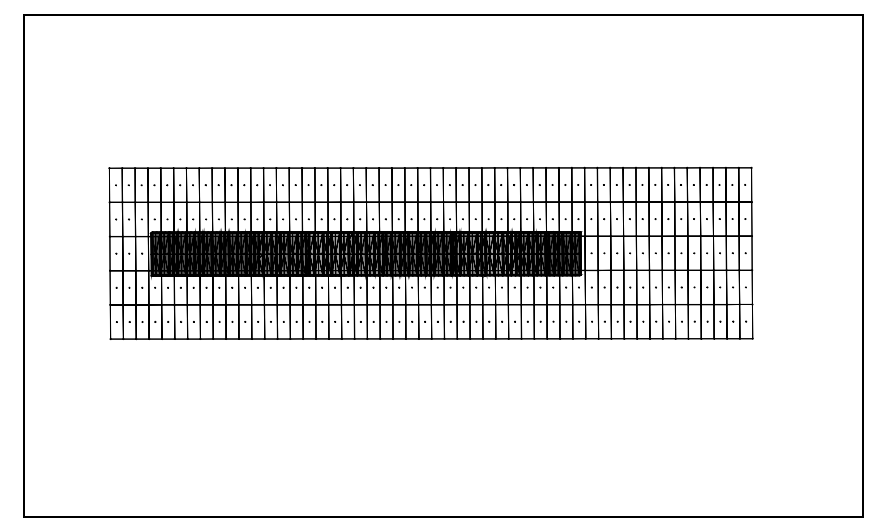

Fig. 4. Top view of a beam over a ground plane example

into 50 parabolic elements and the ground plane is discretized into 2504 -node elements. When a positive potential with reference to the ground plane is applied on the beam, the beam deflects towards the ground plane because of the electrostatic force. As the potential difference increases, the tip of the beam approaches the ground plane, and touches the ground plane for a certain bias defined as the pull-in voltage. The pull-in voltage for the beam considered here is 17.24 volts.

Figure 5 compares the peak deflection obtained from the relaxation, multi-level Newton and coupled algorithms. The results are identical verifying the accuracy of each solver. The deflection of the beam for an applied bias of $17.23 \mathrm{~V}$ is shown in Figure 6.

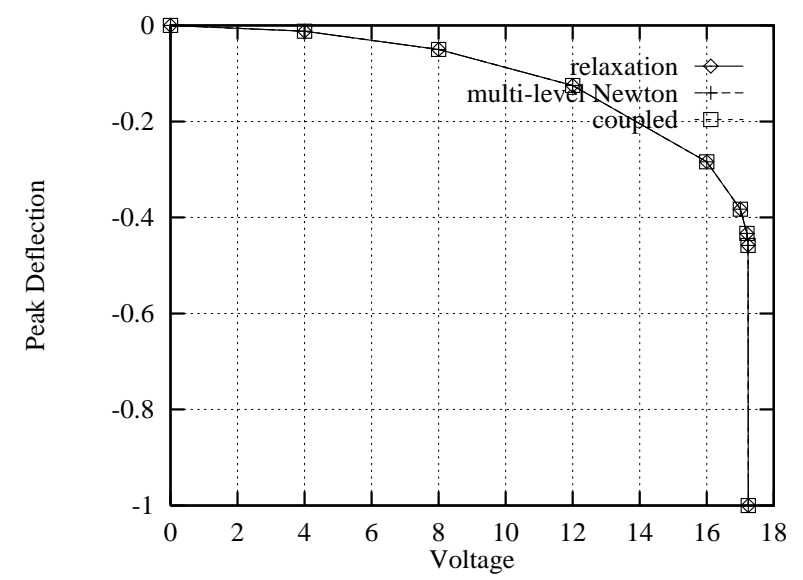

Fig. 5. Comparison of peak deflections from relaxation, multi-level Newton and coupled algorithms for a beam over a ground plane structure. Note that the three curves overlap.

The performance of the relaxation, multi-level Newton and coupled algorithms for the beam example is summarized in Table I. Observe that the multi-level Newton and coupled algorithms take fewer iterations and are much faster compared to the relaxation algorithm for tightly coupled cases. Figure 7 and Figure 8 compare the convergence of the relaxation, multi-level Newton and coupled algorithms for the beam and ground plane example. Note that closer to pull-in the relaxation algorithm converges slowly, but the multi-level Newton and coupled algorithms converge rapidly. The slow convergence of the relaxation algorithm, near pull-in, is due to the increased coupling between elastostatic and electrostatic systems. As the multi-level

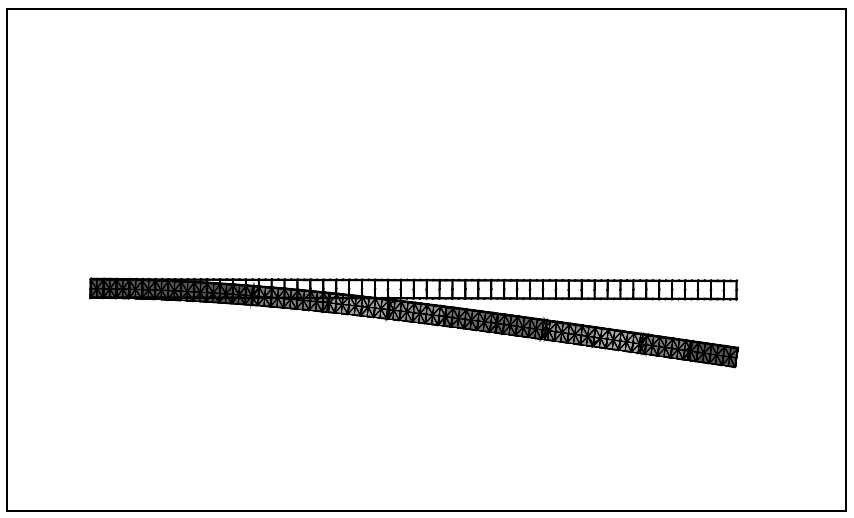

Fig. 6. Deflection of the beam (not to scale) for an applied bias of $17.23 \mathrm{~V}$.

Newton and coupled algorithms accurately account for all the coupling they exhibit rapid convergence behavior. Note also that the multi-level Newton algorithm takes fewer iterations compared to the coupled algorithm, but the coupled algorithm is faster compared to the multi-level Newton technique.

\section{TABLE I}

COMPARISON OF RELAXATION (ALG I), MULTI-LEVEL NEWTON (ALG

II) AND COUPLED (ALG III) ALGORITHMS FOR THE NUMBER OF ITERATIONS AND CPU(SEC) FOR A THICK BEAM AND GROUND PLANE EXAMPLE

\begin{tabular}{|l|c|c|c|c|c|c|}
\hline \multirow{2}{*}{ Bias } & \multicolumn{3}{|c|}{ \# Iterations } & \multicolumn{3}{c|}{ CPU(sec) } \\
\cline { 2 - 7 } & Alg I & Alg II & Alg III & Alg I & Alg II & Alg III \\
\hline 2.0 & 4 & 2 & 2 & 283.5 & 698.7 & 368.4 \\
\hline 4.0 & 5 & 3 & 3 & 381.0 & 967.0 & 476.2 \\
\hline 6.0 & 6 & 3 & 3 & 507.7 & 1244.9 & 514.5 \\
\hline 8.0 & 7 & 3 & 3 & 608.4 & 1079.6 & 572.4 \\
\hline 10.0 & 8 & 3 & 3 & 710.2 & 1086.8 & 612.4 \\
\hline 12.0 & 10 & 3 & 4 & 909.5 & 1086.7 & 801.3 \\
\hline 14.0 & 13 & 4 & 4 & 1244.4 & 1530.7 & 813.4 \\
\hline 16.0 & 20 & 4 & 5 & 2015.8 & 1499.0 & 1096.0 \\
\hline 17.0 & 41 & 5 & 6 & 4248.1 & 1957.0 & 1399.3 \\
\hline 17.20 & 94 & 5 & 6 & 9713.83 & 2145.7 & 1482.5 \\
\hline 17.23 & 200 & 7 & 9 & 20910.5 & 2823.5 & 2289.8 \\
\hline
\end{tabular}

\section{B. Comb Drive Example}

The comb example consists of a deformable comb structure, a drive structure and a ground plane. As shown in Figure 9, the Fshaped finger structure is the comb, the E-shaped finger structure is the drive, and the rectangular shaped structure is the ground plane. The comb is discretized into 172 parabolic elements, the drive is discretized into 144 linear bricks and the ground plane is discretized into 2688 4-node elements. When a positive potential is applied on the drive structure, and zero potential on the comb and the ground plane, the comb structure deforms out of plane. The deformation of the comb structure for an applied bias of 85 volts is shown in Figure 10. Note that only the comb structure deforms and the drive and the ground plane do not move.

A comparison of the relaxation, multi-level Newton and coupled algorithms for the comb example is summarized in Table II. At low 


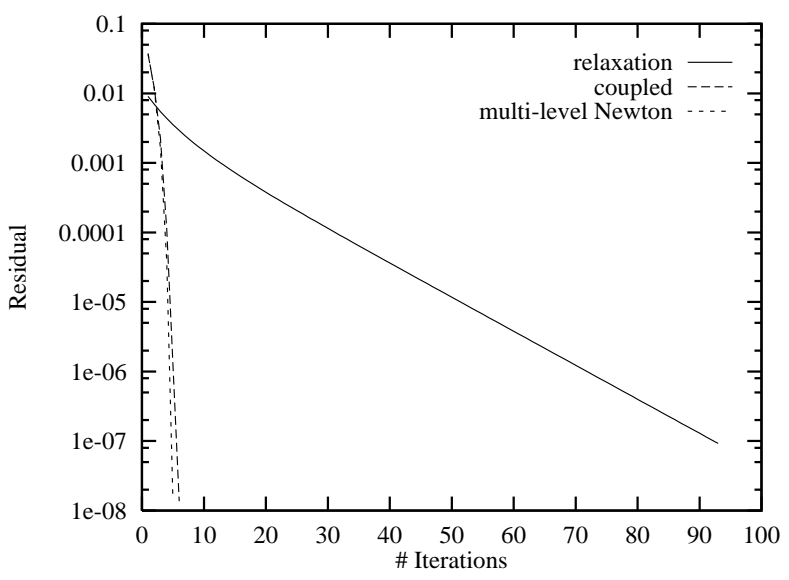

Fig. 7. Convergence of relaxation, multi-level Newton and coupled algorithms for a beam and ground plane structure for an applied bias of 17.20 volts

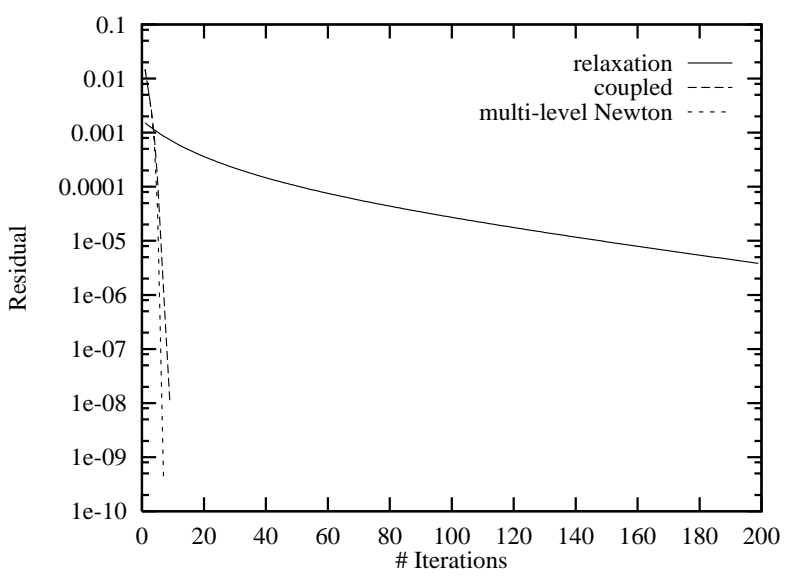

Fig. 8. Convergence of relaxation, multi-level Newton and coupled algorithms for a beam and ground plane structure for an applied bias is 17.23 volts

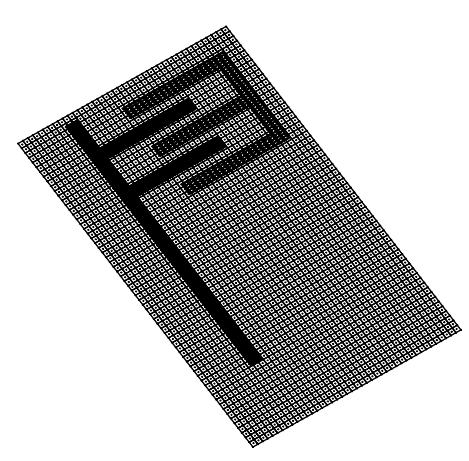

Fig. 9. Comb drive example

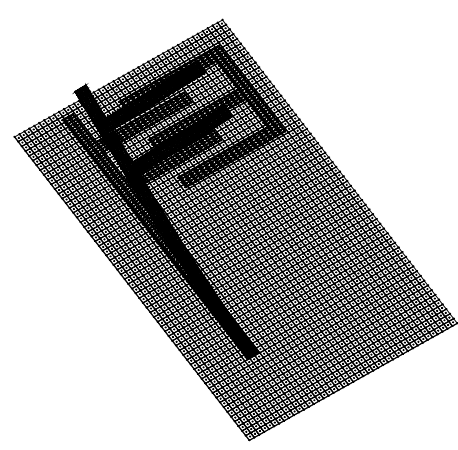

Fig. 10. Deformation of the comb (not to scale) for an applied bias of 85 volts.

voltages, the deflection of the comb is small, the coupling between the electrical and mechanical systems is weak and the relaxation algorithm works very well. At low voltages, both multi-level Newton and coupled algorithms take fewer iterations compared to the relaxation algorithm but the simulation time for both multi-level Newton and coupled algorithms is a little longer. For higher voltages, the multi-level Newton and coupled algorithms converge much faster compared to the relaxation algorithm. For a bias of 80 volts, the multi-level Newton algorithm is about 7.7 times faster and the coupled algorithm is about 5 times faster compared to the relaxation algorithm. The convergence of the relaxation, multi-level Newton and coupled algorithms at 80 $\mathrm{V}$ bias is shown in Figure 11. For an application of $85 \mathrm{~V}$ on the

TABLE II

COMPARISON OF RELAXATION (ALG I), MULTI-LEVEL NEWTON (ALG II) AND COUPLED (ALG III) ALGORITHMS FOR NUMBER OF ITERATION $S$ AND CPU(SEC) FOR A COMB DRIVE EXAMPLE (A * INDICATES THAT THE ALGORITHM FAILS TO CONVERGE FOR THE BIAS)

\begin{tabular}{|c|c|c|c|c|c|c|}
\hline \multirow{2}{*}{ Bias } & \multicolumn{3}{|c|}{ \# Iterations } & \multicolumn{3}{c|}{ CPU(sec) } \\
\cline { 2 - 7 } & Alg I & Alg II & Alg III & Alg I & Alg II & Alg III \\
\hline 25.0 & 7 & 3 & 6 & 3595.4 & 5802.2 & 5589.8 \\
\hline 50.0 & 16 & 4 & 8 & 9138.0 & 10195.1 & 11833.5 \\
\hline 75.0 & 70 & 4 & 10 & 42160.3 & 12053.2 & 18590.7 \\
\hline 80.0 & 142 & 3 & 9 & 81827.0 & 10660.4 & 16670.2 \\
\hline 85.0 & $*$ & 3 & 10 & $*$ & 10767.8 & 18490.9 \\
\hline
\end{tabular}

drive, the relaxation algorithm fails to converge, while the multi-level Newton and coupled algorithms converge very rapidly and take 3 and 10 iterations, respectively. This is illustrated in Figure 12.

\section{CONCLUSION}

In this paper we presented three algorithms for coupled electromechanical analysis. The relaxation technique is a black-box approach but does not converge very well when the electrostatic forces are large or when the structure is very compliant. The full-Newton technique exhibits excellent convergence behavior but it is not a black-box technique. The multilevel-Newton method is a black-box technique and exhibits excellent convergence behavior. For microdevices involving two or more coupled energy domains, the multilevel-Newton technique appears to be the most attractive. CPU results indicate that both 


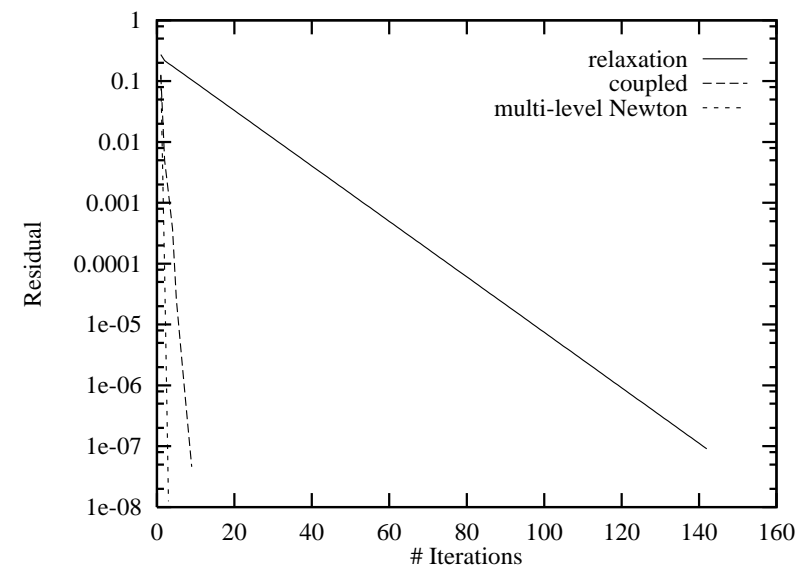

Fig. 11. Comparison of convergence of relaxation, multi-level Newton and coupled algorithms for a comb example at an applied bias of $80 \mathrm{~V}$.

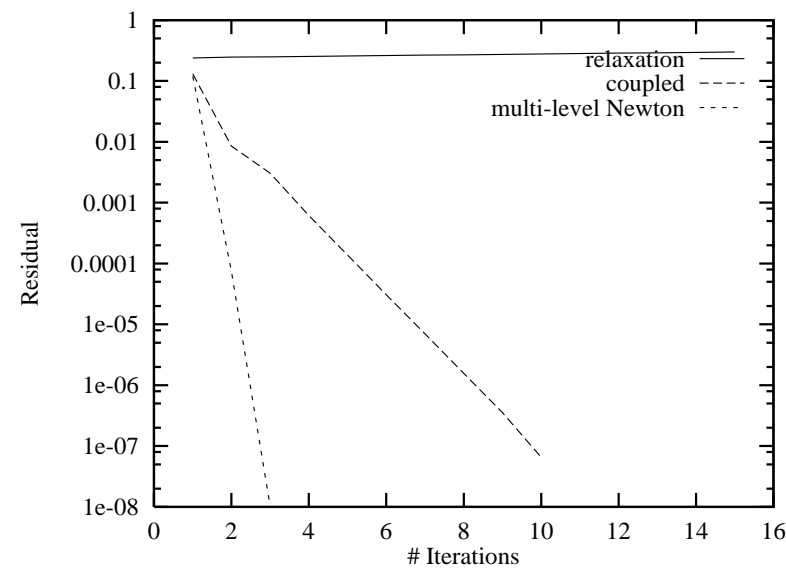

Fig. 12. Comparison of convergence of relaxation, multi-level Newton and coupled algorithms for a comb example at an applied bias of $85 \mathrm{~V}$.

full-Newton and multilevel-Newton are faster techniques compared to relaxation. From our preliminary studies, it is hard to conclude which one of the full-Newton and the multilevel-Newton techniques is a faster approach for microelectromechanical CAD. Several improvements to the full-Newton method are possible and are currently being investigated. A comparison of the three methods with improvements to the full-Newton method should provide better insight in indentifying the most efficient approach.

\section{REFERENCES}

[1] N. Aluru and J. White, "Direct Newton finite-element/boundaryelement technique for micro-electro-mechanical-analysis," Technical Digest, Solid-State Sensor and Actuator Workshop, pp. 54-57, Hilton Head, June 1996.

[2] M. Bachtold, J. G. Korvink, J. Funk and H. Baltes, "New convergence scheme for self-consistent electromechanical analysis of iMEMS," Proc. IEDM 1995.

[3] K. J. Bathe, E. Ramm and E. L. Wilson, "Finite element formulation for large deformation dynamic analysis,"'Int. J. Num. Meth. Engrg., vol. 9, pp. 353-386, 1975.
[4] X. Cai, H. Yie, P. Osterberg, J. Gilbert, S. Senturia and J. White, "A relaxation/multipole-accelerated scheme for self-consistent electromechanical analysis of complex 3-D microelectromechanical structures," Proc. ICCAD 1993.

[5] J. R. Gilbert, R. Legtenberg and S. D. Senturia, "3D coupled electromechanics for MEMS: Applications of CoSolve-EM," Proc. MEMS 1995.

[6] L. E. Malvern, Introduction to the Mechanics of Continuum Medium, Prentice-Hall, Englewood Cliffs, N.J., 1969.

[7] K. Nabors and J. White, "FastCap: A multipole-accelerated 3-D capacitance extraction program," IEEE Trans. CAD, vol. 10, pp. 1447-1459, 1991.

[8] J. R. Phillips and J. White, "A Precorrected-FFT method for capacitance extraction of complicated 3-D structures," Proc. ICCAD 1994.

[9] A. Ruehli and P. A. Brennen, "Efficient capacitance calculations for three-dimensional multiconductor systems," IEEE Trans. Microwave Theory Tech., vol. MTT-21, pp. 76-82, Feb. 1973.

[10] Y. Saad and M. H. Schultz, "GMRES: A generalized minimal residual algorithm for solving nonsymmetric linear systems," SIAM J. Sci. Stat. Comput., vol. 7, pp. 856-869, 1986.

[11] H. U. Schwarzenbach, J. G. Korvink, M. Roos, G. Sartoris, and E. Anderheggen, "A microelectromechanical CAD extension for SESES,” JMEMS, vol. 3, pp. 162-171, 1994.

[12] H. Yie, X. Cai and J. White, "Convergence properties of relaxation versus the surface-Newton generalized-conjugate residual algorithm for self-consistent electromechanical analysis of 3-D micro-electro-mechanical structures" Proc. NUPAD V 1994. 